\title{
MODELLING SOCIOECONOMIC AND HEALTH \\ DETERMINANTS OF HEALTH CARE USE: \\ A SEMIPARAMETRIC APPROACH
}

Jürgen Maurer

143-2007

๑ mea-Mannheim Research Institute for the Economics of Aging

L13, 17_D-68131 Mannheim_Phone +49 621 181-2773/1862_Fax +49 621 181-1863_www.mea.uni-mannheim.de 


\title{
Assessing Horizontal Equity in Medication Treatment Among Elderly Mexicans: Which Socioeconomic Determinants Matter Most?
}

\author{
Jürgen Maurer*
}

Version: July 2007

\begin{abstract}
Many low- and middle-income countries are currently undergoing a dramatic epidemiological transition, with an increasing disease burden due to degenerative noncommunicable diseases. Inexpensive medication treatment often represents a cost-effective means to prevent, control or cure many of these health conditions. Using micro data from the 2001 Mexican Health and Aging Study, we assess horizontal inequity in medication treatment among older Mexicans before the introduction of Popular Health Insurance in Mexico. In doing so, we investigate the role of various dimensions of socioeconomic status for obtaining indicated medication treatment within a comparatively fragmented health care system that features relatively high out-of-pocket expenditures. Our empirical analysis suggests health insurance coverage as a key socioeconomic determinant of indicated medication use with large and statistically significant positive effects on take-up. The effects of insurance status thereby clearly dominate any other possible effects of socioeconomic status on medication treatment. Our results thus highlight the importance of access to reliable health care and comprehensive coverage for rational medication use in the management of degenerative diseases. In light of this evidence, we expect that recent Mexican health care reforms, which expand health insurance coverage to the previously uninsured population, will alleviate socioeconomic gradients in medication treatment among older people in need.
\end{abstract}

Keywords: Medication Treatment, Socioeconomic Status, Equity, Mexico

\footnotetext{
${ }^{*}$ Mannheim Research Institute for the Economics of Aging (MEA), University of Mannheim, L13, 17, 68131 Mannheim, Germany. Email: juergen.maurer@iue.it. I am deeply grateful to Emma Aguila, Emily Sinnott and seminar participant at the MEA and the UNU WIDER conference on "Advancing Health Equity" for valuable comments and suggestions. Remaining errors are, of course, my own responsibility.
} 


\section{Introduction}

Many developing countries are currently experiencing an advancing epidemiological transition away from primarily communicable diseases to a "dual burden" of both infectious and degenerative diseases. In 2001, major degenerative diseases such as cancer, diabetes or heart disease already accounted for more than one-half of all deaths in low- and middle-income countries, with cardiovascular disease alone killing nearly three times as many people as AIDS, malaria, and TB combined (Lopez et al., 2006). The increasing importance of degenerative diseases in low- and middle-income countries calls for new strategies in the delivery of cost-effective health care, which should reflect the changing health care demands implied by the epidemiological transition. In this regard, comprehensive life-style interventions and low-cost disease management programs are often considered promising tools for controlling major risk factors and alleviating the impending burden of degenerative noncommunicable diseases. Such interventions seem particularly well-suited for developing country settings, where health care budgets tend to be especially tight (Jamison et al., 2006a, Jamison et al., 2006b). Yet, to this day, surprisingly little research has been devoted to the health system challenges implied by spreading noncommunicable disease epidemics throughout the developing world (Behrman et al., 2006). Hence, relatively little is known about the main micro-level obstacles impeding the take-up of specific strategies for chronic disease management and risk factor control.

Drug treatments generally constitute an important aspect of comprehensive preventive care strategies, and some of these seem especially well-suited for long-term disease and risk management in a developing country setting. Inexpensive medication treatment often represents an important means to prevent, control or cure many degenerative health conditions. For example, the low cost and high effectiveness of drugs to prevent, say, the reoccurrence of a cardiovascular event is likely to make their long-term use highly cost-effective, even in low- and middle-income countries (Jamison et al., 2006a, Jamison et al., 2006b). Used properly, drugs may also help older people to function better and enhance personal wellbeing by controlling many symptoms associated with various diseases.

Making appropriate medication available to everyone in need irrespective of her socioeconomic status is a central desideratum of an accessible, equitable and responsive health care system. Yet, obstacles such as a lack of relevant health information, high prices coupled with a lack of insurance coverage or insufficient financial resources, or a general lack of access to reliable distribution channels like health centers or pharmacies often thwart the actual take-up of long-term medication treatment for many patients, especially in the developing world.

Mexico can be regarded a case in point with respect to many of these issues. Being an uppermiddle-income country, Mexico is in the fore regarding the shift in the causes of illness in the developing world, as it has undergone a rapid epidemiological transition over the last several decades. Between 1955 and 2004, the proportion of deaths attributable to communicable diseases dropped from $70 \%$ to $12 \%$, whereas the corresponding share of noncommunicable diseases had risen from 23\% to $75 \%$ (González-Pier et al., 2006). Being mostly degenerative illnesses, these noncommunicable diseases are especially concentrated among older individuals. 
Cardiovascular diseases are nowadays by far the leading contributor to premature death in persons age 60+ in Mexico with an associated mortality rate more than four times higher than that of all communicable diseases (PAHO, 2002). Morbidity has changed similarly, with many older Mexicans now suffering from several, often chronic health conditions (Palloni et al., 2004). Estimated prevalence rates for conditions such as hypertension or arthritis amongst individuals 60 years of age and older are well above $20 \%$, with the corresponding rate for diabetes being close to that level.

Apart from Mexico's typical epidemiological profile, some institutional characteristics of the Mexican health care system make it also an interesting case to study. Prior to recent health care reforms, Mexico's health system could be characterized as largely fragmented and underfunded with fairly incomplete insurance coverage for the population at risk. In 2000, around one-half of the Mexican population was without publicly funded health care insurance which resulted in comparatively low levels of overall health spending, an elevated share of out-of pocket spending as well as unequal public resource allocation between the socially insured and those with no social security coverage (González-Pier et al., 2006). The coexistence of public, private and social security subsystems with social security coverage limited to workers in the formal sector is in fact a quite common characteristic of many health care systems in other low- and middle income countries, especially in Latin America (Cetrángolo et al., 2006). The policy challenges implied by such a largely fragmented health care system are therefore not exclusive to Mexico.

To remedy the lack of accessibility, equity and responsiveness as well as the occurrence of excessive out-of-pocket spending on health care, the reform of the Mexican General Health Law established a System of Social Protection in Health in 2004, including the introduction of a new scheme of Popular Health Insurance (Seguro Popular de Salud). The Seguro Popular aims at promoting universal access to social protection in health, by providing subsidized insurance to the previously uninsured, which covers a package of essential health care interventions, including essential medication treatment. It is currently still in its implementation phase, with complete rollout expected to be completed by 2010 (Gakidou et al., 2006, Knaul and Frenk, 2005). ${ }^{1}$

The present study takes a closer look at socioeconomic inequity in medication treatment in Mexico prior to the introduction of Seguro Popular. Using data from the 2001 Mexican Health and Aging Study (MHAS), we assess the impact of various dimensions of socioeconomic status on indicated drug use of older Mexicans among whom degenerative diseases are naturally concentrated.

The purpose of the paper is twofold. On the one hand, the present study investigates the socioeconomic determinants of access to medication treatment - an important component of chronic disease management - in a middle-income setting. Using Mexico as a typical example for a fragmented and underfunded health care system, the study assesses to what extent older patients are able to obtain the medicines they need. In doing so, we also identify which socioeconomic characteristics appear most important for achieving treatment take-up. We thus

\footnotetext{
${ }^{1}$ See also Gakidou et al. (2006) for an interim evaluation.
} 
study the respective role of various dimensions of socioeconomic status to overcome the obstacles implied by a fragmented and underfunded health care system that lacks comprehensive coverage for the population at risk.

Apart from any potentially useful global lessons, the paper also presents evidence relevant to the Mexican health policy debate. Particularly, we identify to what extent there were any gaps in the take-up of medication treatment prior to the introduction of the Seguro Popular and how these were related to various dimensions of socioeconomic status. Highlighting the role of insurance status for medication use, our study also provides an ex-ante assessment of the likely effects that a successful implementation of the Seguro Popular will have on the take-up of drug treatment among the previously uninsured. It thus allows us to gauge the likely demand response implied by the rollout of this new insurance scheme.

The remainder of the paper is organized as follows: Section 2 gives some more detailed background information on the Mexican health care system, particularly as it pertains to the availability and coverage of medication treatment for the chronically ill. Section 3 briefly reviews our conceptual framework, highlighting the main ideas underlying our empirical model. Section 4 introduces the data and gives some details on the exact measures used in the analysis. Section 5 presents our estimation results. In conclusion, section 6 gives a summary of the main results as well as a discussion of some potential caveats and the most important policy implications of the paper.

\section{The Mexican Health Care and Pharmaceuticals Market in a Nutshell}

This section briefly highlights some institutional characteristics of the Mexican health care system with respect to health insurance coverage and access to care. We also present a short review of how these institutional features affect the coverage of pharmaceuticals and their delivery to patients. ${ }^{2}$

\subsection{The Mexican Health Care System}

Prior to the introduction of the Seguro Popular in 2004, the Mexican health care system was widely regarded as comparatively fragmented, underfunded and inequitable. In Mexico, large social insurance institutions provide health care for formally employed workers and their relatives, for whom social insurance is compulsory. Formal workers of private companies are typically covered by the largest social insurer, the Instituto Mexicano del Seguro Social (IMSS, Mexican Social Security Institute); while government employees are covered by the Instituto de Seguridad y Servicios Sociales de los Trabajadores del Estado (ISSSTE, Institute of Social Security and Services for Government Workers). The major social security agencies operate

\footnotetext{
${ }^{2} \mathrm{~A}$ full description of the Mexican health care system is beyond the scope of this paper. OECD (2005a) gives a more complete overview the Mexican health care system, while Moise and Docteur (2007) provide an extensive review of the pharmaceutical market in Mexico.
} 
their own health facilities, which can be used by their beneficiaries mostly free of charge. Social insurance coverage does therefore not simply imply reimbursement for health care spending, but affects health care delivery directly through the default provider. In 2001 - the base year for our analysis - IMSS insured more than 45 million Mexicans, with a further 10 million government employees covered by the ISSSTE; IMSS and ISSSTE together covered around 95\% of Mexico's socially insured population, with the remaining 5\% being insured via smaller social security agencies, which include the navy (NAVY), the Ministry of Defense (DEFENSE) and Mexico's state-owned oil company, Petroleos Mexicanos (PEMEX) among others.

Private health insurance has not been very widespread in Mexico. Just around $3 \%$ of the population had any form of private health insurance and roughly half of that was accounted for by employer subsidized group plans. To expand coverage, the government introduced two voluntary health insurance programs in 1997, the Incorporacion Voluntaria al Regimen Obligatorio (Voluntary Incorporation to the Obligatory Regime) for the self-employed and the Seguro de Salud para la Familia (Health Security for the Family) for the workers in the informal sector. Yet, registration into these programs has been minimal, as it has generally been considered too expensive to buy into (Moise and Docteur, 2007).

Despite the fact that IMSS and ISSSTE offer free services to their beneficiaries, the private medical care market has not been only used by the uninsured. Rather, some social security beneficiaries also relied on the private sector for complementary service delivery. In fact, the private sector encompasses many service providers of differing quality, ranging from specialtytrained physicians and high-technology medical centers serving the wealthy to unregulated and unsupervised private physicians without specific training that deliver health care mostly to the uninsured (Barraza-Lloréns et al., 2002).

In sum, just over half the Mexican population had any health insurance coverage prior to the 2004 reform, mostly tied to formal employment. The other half of the population, i.e. those outside the formal sector, generally had no insurance coverage, as the social insurers only covered formally employed workers and their relatives and other affordable forms of health insurance were generally unavailable (OECD, 2005a). The uninsured mainly relied on state health care services through the ministry of health which were generally subject to a socioeconomic evaluation at the time of treatment and may have charged some user fees. Alternatively, the uninsured could seek care in the private medical care market which was generally for-profit, charging fees for service. The fragmented nature of the Mexican health care system as well as its lack of comprehensive insurance coverage resulted in a considerable degree of inequity, with both a high incidence of out-of-pocket health care spending as well as a general underutilization of services concentrated among the uninsured (OECD, 2005a). ${ }^{3}$

To remedy the disadvantaged situation of the uninsured, the reform of the General Health Law, which came into effect in January 2004, established - amongst other things - the Seguro

\footnotetext{
${ }^{3}$ More than $50 \%$ of health care spending was directly financed by households and went into the private sector. As a consequence of the general lack of affordable private health insurance, more than $95 \%$ of these expenditures where financed through direct out of pocket payments and less than $5 \%$ through prepaid insurance (Barraza-Lloréns et al., 2002).
} 
Popular. The Seguro Popular will provide health services and cover essential medical care as well as some high-complexity interventions for all uninsured households after a legally mandated seven year phase-in period (Gakidou et al., 2006). It is expected to be fully in effect by 2010 . The Seguro Popular is publicly subsidized, but also requires a means-tested annual payment from affiliated families, with an exemption for families in the lower two income deciles. In addition to providing some financial protection for the previously uninsured, it is also hoped that the Seguro Popular will alleviate underutilization of health care and help to increase care use among those in need.

\subsection{Pharmaceutical Coverage and Delivery in Mexico}

The fragmented and underfunded nature of the Mexican health care system has affected all forms of care delivery, including the coverage of pharmaceuticals and their delivery to patients.

On the one hand, social insurance beneficiaries can obtain most prescription drugs free of charge, as long as they are obtained from an agency-run pharmacy. Thus, the social insurers do not generally require any cost-sharing for prescribed medication covered by their respective formularies. ${ }^{4}$ Yet, some social security beneficiaries nonetheless chose to buy their medication in private pharmacies at full cost, as they may otherwise have to wait for the drugs to become available in their provider pharmacies or may not get the brand type they wanted (Moise and Docteur, 2007).

The uninsured, on the other hand, have mainly had to rely on purchasing their medication from private pharmacies. While drugs used for inpatient treatment in Ministry of Health or state health service facilities have been generally free of charge, prerefrom the uninsured patients had to pay out-of-pocket for continued drug treatment after discharge, even with regard to the same care episode. Other medication, like the ones used for chronic disease management, also had to be bought at private pharmacies and paid for out-of-pocket. Overall, these arrangements have led to a very large share for out-of-pocket spending on medications, comprising around 90\% (OECD, 2005b) of total pharmaceutical spending in Mexico.

Access barriers to health care in general and incomplete coverage of pharmaceuticals in particular posed serious threats to rational medication use, especially for the uninsured. Specifically, self-medication has become a widespread phenomenon in Mexico. Pagán et al. (2006) show that self-medication was prevalent in all socioeconomic strata of the population, but most widespread among individuals with no health insurance coverage. On the one hand, self-medication may in fact be beneficial, as it may enhance the take-up of medication treatment among patients with no access to primary care (WHO, 2000). On the other hand, using pharmaceuticals without prior consultation with a doctor may also result in poor diseasemedication-matches and inappropriate drug use that does not accurately reflect a patient's true health care needs. Similarly, lack of coverage may result in inappropriate substitution

\footnotetext{
${ }^{4}$ Note that the formularies of the two major insurers, IMSS and ISSSTE, are fairly similar, with all listed drugs being also included in the interinstitutional basic formulary, the Cuadro Basico (Basic Coverage)(Moise and Docteur, 2007).
} 
between drugs and is likely to lead to no and/or incomplete take-up of indicated medication treatments. Moreover, such forms of underutilization are likely to be further exacerbated by the comparatively high income-adjusted price-level of pharmaceuticals in Mexico (Danzon and Furukawa, 2003, Ministry of Health, 2005). The problem of underutilization is then particularly relevant for the case of risk factor control. Many risk factors, such as hypertension, are largely asymptomatic and may thus result in low perceived health care need.

In addition to covering a package of 249 essential and 17 high-complexity health interventions (González-Pier et al., 2006), the Seguro Popular also provides coverage for almost 200 pharmaceuticals, with the list of essential medicines being continuously revised. ${ }^{5}$ As such, the Seguro Popular will not only enhance access to primary and secondary health care, but also facilitate the use of pharmaceuticals for disease management and risk factor control. It is therefore interesting to investigate to what extend the uninsured were able to use alternative strategies for take-up, such as self-medication, before the introduction of the Seguro Popular. Also, assessing the partial effects of insurance status on indicated medication use will allow us to gauge the likely effects of the Seguro Popular on take-up for the previously uninsured.

\section{Conceptual Framework and Control Variables}

We use a simple version of the behavioral model of health care utilization as the basis for our empirical analysis of horizontal inequity in medication treatment (Andersen, 1968, Andersen, 1995). The behavioral model features a clear distinction between care need on the one hand and enabling factors on the other. Assessing the extent of socioeconomic inequity in medication use thus requires an appropriate need-adjustment. Specifically, we would like to identify the importance of different socioeconomic enabling factors for the take-up of medication treatment only for those who do require such treatment. ${ }^{6}$ We therefore have to condition on the respondents' background health in order to properly identify potential gaps in beneficial medication treatment. To do so, we confine our analysis to individuals with self-reported doctor-diagnosed health conditions that warrant some form of medication treatment.

This strategy resembles the disease-based approaches that have been recently suggested by the OECD among others (Goldman and Smith, 2005, Jacobzone, 2003, Merino-Juárez, 2004). In the Mexican context, the disease-based approach appears fairly conservative. Specifically, conditioning on doctor-diagnosed health conditions will - if anything - understate the extent of a possible socioeconomic gradient in the use of pharmaceuticals. The reason for this is that any preexisting gradients in access to general health care, testing or screening, which might affect the availability of a diagnosis, tend to lead to higher rates of undiagnosed, and thus untreated conditions among disadvantaged groups. ${ }^{7}$ Similarly, such gradients make it also more likely

\footnotetext{
${ }^{5}$ http://www.salud.gob.mx/unidades/dgpfs/catalogo_medicamentos.htm (accessed on 10/07/2007).

${ }^{6}$ See also Wagstaff and van Doorslaer (2000) regarding the treatment of "need" vs. "non-need" determinants of health care utilization in health equity analysis.

${ }^{7}$ Pagán et al. (2007) show that such preexisting gradients may indeed be important, as individuals with social health insurance coverage are much more likely to use preventive screenings that individuals without insurance.
} 
that among the disadvantaged, only the more severe causes obtain a diagnosis and thus feature in our estimation samples. Both of these issues suggest a lower bound interpretation of any estimated socioeconomic gradients in medication use conditional on having some diagnosis. Over and above conditioning on a reported diagnosis, we also include a set of age controls into our empirical model to account for the progressive nature of some of these conditions. Finally, we also account for comorbidities and potentially important gender differences in the determination of care need.

Turning to the enabling factors, we aim at capturing various dimensions of socioeconomic status to assess which socioeconomic determinants matter most for the take-up of indicated medication use. Our first enabling factor intends to capture potentially important differences in drug use between rural and urban areas. It is well-known that there are vast regional differences in health system performance in Mexico, which are also likely to affect the availability and use of pharmaceuticals. Specifically, as access to health care is clearly related to the available health infrastructure such as resident doctors, hospitals and pharmacies, it is essential to control for differences between rural and urban settings in our empirical model.

Our second enabling factor captures individual differences in human capital, which is likely to affect a patient's capacity to assess and evaluate the benefits of medication treatment. We thus consider human capital as a potentially important determinant for (self-) medication takeup and treatment compliance, even if the available evidence from other countries is mixed (Case et al., 2004; Goldman and Smith, 2002, 2005; Lleras-Muney and Lichtenberg, 2002).

Our third set of enabling factors is mainly motivated by the large share of out-of-pocket expenditures on pharmaceuticals in Mexico. Specifically, we include controls for both income and wealth in our model to capture potentially important differences in individuals' capacities to pay for medication out-of-pocket.

The final set of socioeconomic controls directly relates to the institutional arrangements of the Mexican health system, namely whether the respondent has any social insurance coverage. The potential effects of insurance coverage on the take-up of medication treatment may thereby operate through various channels, including better access to primary care and screening programs, higher care quality and better disease monitoring as well as more generous prescription benefits, such as free prescription medication from agency-run pharmacies. Highlighting the importance of insurance coverage relative to other enabling factors such as income or wealth may provide some additional insights on the likely effectiveness of introducing governmentsponsored health insurance, such as the Seguro Popular, for ensuring adequate medication treatment for the previously uninsured.

Having health insurance coverage is often suspected to be endogenous with respect to health care use. Yet, as highlighted previously, there has basically not been a market for private health insurance in Mexico, and social insurance coverage used to be closely tied to formal employment, in which case it is compulsory. As such, Mexican workers have usually not had the choice to directly buy into health insurance. Rather, the relevant decision used to be whether to formalize or not, which is largely influenced by a worker's labor market opportunities as well as other 
considerations related to social protection more generally, such as unemployment insurance or pensions, as well as to the financing of coverage, which has included some payroll taxes on part of the workers (Perry et al., 2007). Hence, the decision of whether or not to obtain any health insurance coverage is only indirectly under the respondents' control. Beyond it, the absence of choice with respect to take-up of medical insurance is even more pronounced among the older population. The reason for this is that most older individuals are insured through a relative, such as a spouse, daughter or son, who in turn, face the decision on whether or not to formalize rather than to just take-up health insurance. Given the lack of direct control over one's insurance status and the absence of an obvious instrument for the decision to formalize, we abstract from the potential endogeneity of health insurance status and treat it as exogenous.

\section{Data and Measures}

\subsection{Data}

Our empirical analysis is based on data from the first wave of the Mexican Health and Aging Study (MHAS) collected in 2001. The MHAS is a nationally representative sample of the non-institutionalized older Mexican population (age 50+) and their spouses, the latter being included in the study regardless of their age. Similar in design to the US Health and Retirement Study, the MHAS collects extensive demographic, economic and health-related information. The MHAS thus represents an ideal data source for analyzing any socioeconomic differentials in health care utilization. For our analysis, we only use observations on individuals aged 40 or older who have no missing item on any of the variables employed $(\mathrm{N}=14397){ }^{8}$

\subsection{Measures}

\subsubsection{Outcomes of Interest}

We analyze the effects of socioeconomic status on the take-up of medication treatment conditional on care need. To account for the latter, we select respondents who reported to have been doctor-diagnosed for at least one out of the following six conditions: Arthritis, diabetes, heart attack, hypertension, respiratory disease or stroke. In the estimations, we thus only considered respondents with health conditions for which drug treatment is generally believed to be beneficial. For arthritis, the use of nonsteroidal anti-inflammatory drugs, slow-acting drugs, corticosteroids or immunosuppressive drugs is often indicated. Diabetics, on the other hand, generally need to rely on antihyperglycemic drugs or insulin replacement therapy if dieting and exercise appear to be insufficient to control the blood sugar level. We thus enquired whether those with self-reported doctor-diagnosed diabetes used either antihyperglycemic drugs or insulin or both. For heart attack patients, we also consider two forms of medication treatment.

\footnotetext{
${ }^{8}$ Note that the deletion of observations due to item non-response was performed after incorporating the MHAS-provided imputations for total individual income and total household wealth (see Wong and Espinoza (2004) for methodological details regarding the imputations).
} 
Firstly, we code the use of any heart medication such as nitroglycerin, beta-blockers, ACE inhibitors, and lipid-lowering drugs, which are generally indicated following a heart attack, as any drug use among heart attack patients. In addition, we also count patients as using medication treatment if they reported carrying any medication for heart/chest pain, such as aspirin, which substantially increases the probability of survival in case of a further heart attack. For respondents with hypertension, we investigate the use of antihypertensive drugs to control blood pressure. The next health conditions we consider are respiratory diseases such as asthma or emphysema for which the use of anti-inflammatory drugs such as corticosteroids is often indicated. Finally, we consider respondents with a doctor-diagnosed stroke or transient ischemic attack. For those, the use of antiplatelet drugs is generally useful to prevent further onsets of such conditions.

Given this classification of patients as treated or untreated, we follow two distinct approaches for our empirical analysis. The first approach is based on a pooled sample of all respondents indicating at least one health condition. For this sample, we assess the extent of socioeconomic differences in medication use based on two different concepts of treatment take-up. On the one hand, we investigate whether the respondents used any medication for at least one of their conditions ("any use"), capturing the notion of "access" to any medication treatment. On the other hand, we also assess whether or not these respondents reported "any gap" in medication treatment. We thereby define "any gap" as reporting at least one untreated health condition. The second concept therefore gauges the extent of "incomplete take-up" of medication treatment.

The second approach is truly disease-based in the sense that we consider only one disease at a time. Specifically, we estimate models of medication use for each of the above six health conditions separately, where the corresponding estimation samples only include respondents that indicate the respective condition under consideration. Estimating such disease-specific models has the advantage that the two concepts of "any use" and "any gap" collapse into a single variable, namely whether the condition under consideration is medicated or not. Depending on the prevalence rate of the respective health condition at hand, the increased specificity of the disease-based approach results in much smaller sample sizes, though, which are likely pose a challenge with respect to estimation precision.

Table I presents sample means for the indicators defining our sample selections as well as all of the associated outcome measures. Around $55 \%$ of all MHAS respondents reported suffering from at least one doctor-diagnosed health condition, leading to a pooled estimation sample of size $\mathrm{N}=7917$. Among these, $74 \%$ reported using medication for at least one of their health conditions ("any use"). At the same time, $40 \%$ of those respondents reported at least one untreated health condition ("any gap"), i.e. less than complete take-up of medication treatment. Table I also gives a more detailed breakdown by disease: $19 \%$ of the MHAS respondents reported a doctor-diagnosed arthritis, resulting in a disease-specific estimation sample of $\mathrm{N}=2755$ respondents. Out of those, $58 \%$ stated some drug use to treat their arthritis. Similarly, $15 \%$ of the full sample reported suffering from diabetes $(\mathrm{N}=2181)$, with $86 \%$ percent 
of those indicating the use of either antihyperglycemic drugs or insulin replacement therapy. The prevalence rate of heart attacks, on the other hand, is fairly small with only $3.3 \%$ of the full sample $(\mathrm{N}=473)$ reporting a corresponding diagnosis. Among those, $71 \%$ also reported using some drug for their heart condition. The condition with the highest prevalence rate is hypertension $(37 \%, \mathrm{~N}=5307)$, with an associated take-up rate of $71 \%$ for antihypertensive drugs. Finally, around $6 \%$ of the respondents reported some respiratory condition $(\mathrm{N}=872)$, and $2.6 \%$ reported a stroke $(\mathrm{N}=370)$, with around one half of those respondents reporting some medication treatment for their respective condition ( $47 \%$ and $50 \%$, respectively).

Altogether, more than one half of the older Mexican population report some health condition, with a substantial fraction also indicating no or less than complete take-up of medication treatment. The prevalence rates for the various health conditions vary substantially within our sample, with chronic diseases being considerably more prevalent than acute events survived. Interestingly, the take-up rates of medication treatment feature substantial variation, too, ranging from fairly comprehensive take-up of diabetes treatment to comparatively low drug use for respiratory conditions.

\subsubsection{Control Variables}

Following the conceptual framework outlined in the previous section, our set of control variables can be broadly grouped into two categories, aligned with health care need on the one hand and enabling factors on the other. Regarding the former, our estimations include dummy variables for ten year age bands (50-59, 60-69 and 70+, with 40-49 being the omitted category) to account for the progressive nature of some of the underlying conditions. We also construct a dummy variable indicating the presence of multiple conditions to control for comorbidities.

Moreover, all of our empirical models also include a gender dummy. Gender is in fact a hard case for categorization within the simple dichotomy of need vs. enabling factors suggested by the behavioral model. Clearly, gender may capture some underlying biological differences related to the severity of a specific health condition and thus be a need indicator. At the same time, gender also constitutes an important aspect of socioeconomic status and may thus be counted as an enabling factor, too. Given these difficulties regarding the categorization of gender, we include it for need-adjustment in all of our models, but also report its estimated partial effects along with those of the other controls classified as enabling factors.

In this regard, the previous discussion has highlighted urban vs. rural environments, human capital, financial resources and insurance status as potentially important socioeconomic determinants of medication use. In terms of measurement, our models include two indicator variables for the size of the respondent's residence. Specifically, we control for whether the respondent lives in a city of medium size (15000-100000 inhabitants) or in a bigger city (more than 100000 inhabitant), with rural areas (1-14999 inhabitant) forming the omitted category. To control for the effects of human capital, we construct four education categories - roughly corresponding to the quartiles of completed years of schooling in the full sample - which indicate whether the respondent has spent 2-3 years, 4-6 years or more than 6 years in school 
(with no or 1 year of schooling being the omitted category). Similarly, we construct indicator variables for the respondents' income and wealth quartile. Finally, we constructed a dummy variable indicating whether the respondent has any health insurance. This indicator variable takes on a value of one if the respondent reports coverage under at least one of the following schemes, IMSS, ISSSTE, NAVY/DEFENCE/PEMEX, private insurance or any other type of health insurance, and is zero otherwise. In addition, we also constructed an indicator for multiple insurance coverage, i.e. whether or not a respondent is covered by more than one of the aforementioned schemes.

Table II presents sample means for all control variables used in the analysis for the full as well as all estimation samples. By and large, our estimation samples seem quite similar to the overall MHAS sample, although some minor differences emerge. As one would expect, the estimation samples tend to be somewhat older than the full sample. This reflects the fact that we consider mostly degenerative conditions, whose prevalence rates naturally increase with age. Also, the estimation samples mirror well-known gender patterns of disease, with a somewhat higher relative share of women in all disease categories, but heart attack and stroke. Regarding our socioeconomic controls, the estimation samples are slightly more urban, somewhat less educated, and have slightly lower income and wealth. At the same time, the estimation samples include more respondents with any health insurance coverage. Concerning this matter, the table also presents a more detailed account of the specific types of health insurance underlying our binary insurance indicator. IMSS is clearly the most widespread type of health insurance, covering around $50 \%$ of the respondents in the various samples. ISSSTE, in turn, covers around $15 \%$ of the respondents. The remaining categories, NAVY/DEFENCE/PEMEX, private health insurance and other health insurance are all comparatively small, each covering around 2-3\% of the samples. Altogether, around $60-75 \%$ of the respondents report to have some form health insurance coverage, with the exact fraction depending on the respective estimation sample. Finally, around $5-7 \%$ of the respondents have the right to medical attention under more than one insurance scheme.

\section{Results}

\subsection{Pooled Regressions}

We start by investigating the individual associations of our different enabling factors with the take-up of (any or complete) medication treatment conditional on need. We first assess the extent of any partial gradients along the various socioeconomic dimensions considered here, before moving to a full model that includes all enabling factors as control variables.

Table III reports the estimated average partial effects of corresponding probit models. Columns (1)-(6) present the results for any medication use. Specifically, columns (1)-(5) give the individual associations of each enabling factor separately, whereas column (6) presents the results for the full model with all controls.

A first noteworthy feature of all the models is that women are significantly more likely to 
take up medication treatment, with estimated partial effects in the order of 5-6 percentage points.

The individual associations of the pure enabling factors with the need-adjusted take-up of medication treatment all highlight significant gradients in medication use along each dimension of socioeconomic status. When considered individually, take-up of medication treatment is monotonically increasing in each enabling factor and the estimated partial effects are generally statistically significant. Wald-tests for the joint significance of each individual set of dummy variables always lead to clear rejections of the hypothesis of "no effect" with associated pvalues far below .01. Moving from a rural to an urban environment increases the probability of take-up of medication treatment by more than 11 percentage points. Moving from the first to the fourth quartile of educational attainment, in turn, is associated with a 10.4 percentage point increase in medication use. Similar gradients are also found along the distributions of income and wealth, albeit somewhat less pronounced. Ranging from the first to the fourth quartile of income and wealth increases the take-up of medication treatment by 5.3 and 6.6 percentage points, respectively. Finally, having any form of health insurance is associated with a 13 percentage points higher take-up rate, whereas multiple insurance coverage adds little, if anything, to the conditional probability of medication use. Specifically, while the corresponding point estimate on multiple coverage indicates an increased take-up of around 3.5 percentage points, it is not statistically significant at conventional levels. In sum, we find fairly uniform socioeconomic gradients along each individual dimension covered by our enabling factors.

We now move to the full model of column (6), which includes all socioeconomic controls at once. Firstly, women remain significantly more likely to take-up any medication treatment, even after controlling for all other enabling factors. Specifically, we estimate a statistically significant partial effect of being female of around 6 percentage points. Also, moving from a rural to an urban setting still increases the probability of take-up, although the size of the estimated effect reduces substantially. Conditional on all other controls, medication treatment is around 5 percentage points more likely in bigger cities than in small villages. A Wald-test for the joint significance of the two city size controls leads to a p-value of 0.001 , clearly rejecting the null of "no city size effects" at any conventional level.

Similarly, education gradients seem to persist, too, but their size also reduces by around one-half. The corresponding partial effect of moving from the first to the fourth quartile of educational attainment is now estimated to be around 4.6 percentage points. A test for joint significance clearly rejects the null of "no education gradients", with a corresponding p-value of 0.0000 .

Interestingly, none of our income controls remains significant in the full model. While respondents in the 2nd to 4th income quartile are still somewhat more likely to take-up drug treatment, the corresponding partial effects are very small, between 1 and 2 percentage points only. Furthermore, we can also not reject the null of "no income effects" at any conventional levels of significance (p-value: 0.3692).

The dummies for the 3rd and 4th quartile of household wealth, on the other hand, still 
enter the model significantly with estimated partial effects of 3.1 and 3.5 percentage points, respectively. Also, with a p-value of 0.0169 , we can reject the null hypothesis of "no wealth gradients" in take-up, albeit only at the $5 \%$-level.

Our last set of enabling factors pertains to the health insurance status of the respondents. The effect of having any health insurance coverage reduces from 13 percentage points in the model with insurance only to around 10 percentage points in the full model. Thus, the estimated effect of insurance coverage on the take-up of medication treatment remains fairly large and is strongly significant. In fact, the estimated partial effect of having some form of insurance coverage is almost twice as large as the second largest effect of any other of our socioeconomic control (living in a city with more than 100000 inhabitants). As before, having multiple insurance coverage does not add much to the probability of take-up and the estimated effect is not statistically significant.

We can now turn to our assessment of "any gaps" in the take-up of medication treatment, estimating conditional probabilities for reporting at least one untreated condition (columns (7)-(12)). Looking at such defined gaps in medication treatment, we find negative factorspecific gradients in the probability of "incomplete take-up" with respect to all enabling factors (columns (7)-(11)). As in the case of any medication use, all estimated gradients are also monotonic in the respective enabling factor under consideration. ${ }^{9}$ Individuals living in urban areas tend to have a lower probability of having any gap in medication treatment, as do the better educated, those with higher income and wealth and those with any health insurance coverage. The factor-specific individual gradients are again most pronounced for city size, education and insurance status, and all sets of enabling factors, but income, are jointly significant at the $1 \%$ level. Even income, however, enters the model significantly at the 5\%-level (p-value of 0.0278$)$.

The results for the full model in column (12) portray a similar picture to the one obtained when analyzing any medication use: The size of the gradient with respect to each enabling factor reduces relative to the factor-specific gradients, but remains fairly sizable and statistically significant for city size ( $\mathrm{p}$-value: 0.0107), education ( $\mathrm{p}$-value: 0.0000), and health insurance status (p-value: 0.0000). The effects of income and wealth are again much less pronounced, and both sets of controls do not enter the model significantly, with corresponding p-values of 0.4424 and 0.1594 , respectively. Among all enabling factors, health insurance coverage has again the largest effect on take-up. Its estimated partial effect of -12 percentage points is almost twice as large as the second largest effect of any socioeconomic control (3rd and 4th quartile of educational attainment).

Altogether, we find coherent evidence for important socioeconomic gradients in the take-up of medication treatment based on a pooled estimation sample of respondents indicating some form of care need. While the data reveal significant monotonic factor-specific gradients for all enabling factors under considerations, our full model, which includes all these factors at

\footnotetext{
${ }^{9}$ Note also, that women have a lower probability of having any gap in medication treatment, which again indicates more medication use among females.
} 
once, identified city size, education, and especially insurance status as the most important socioeconomic determinants for increased take-up of (any or complete) medication treatment.

\subsection{Disease-specific Analysis}

We now proceed by adopting a truly disease-based approach to refine our results and investigate their robustness across different health conditions. Table IV presents estimated partial effects for six full models that include all enabling factors, one separate model for each of the six health conditions.

For most of our socioeconomic controls, the disease-specific regressions portray a less clearcut picture than the pooled analysis. For example, while women are significantly more likely to take-up medication treatment for some chronic diseases such as arthritis, diabetes and hypertension, we do not find any statistically significant effects for gender for respondents reporting a heart attack or respiratory disease, and a fairly large, negative and statistically significant effect for respondents reporting stroke.

Similarly, we do also not find an entirely uniform picture for the effects of city size. Most of the estimated partial effects are insignificant with point estimates indicating higher or lower take-up depending on the particular condition under consideration. Yet, whenever city size enters the model significantly - as in the cases of arthritis (p-value: 0.0004) and diabetes (pvalue: 0.001$)$ - the estimated partial effects indicate higher take-up rates in larger municipalities, at least relative to the rural baseline category.

Also, the estimation results for educational attainment display a mixed pattern, rendering it difficult to draw strong conclusions. However, whenever our set of education controls enter the model significantly, that is for arthritis (p-value: 0.0412) and hypertension (p-value: 0.0019 ) and at the 10\%-level for diabetes (p-value: 0.0980) and stroke (p-value: 0.077$)$, the estimated effects are always positive and in some cases fairly large in size. For example, being in the highest quartile of the education distribution increases the conditional probability for the takeup of stroke treatment by 20 percentage points relative to the 1st education quartile.

Interestingly, our set of income controls do not enter any model significantly, with the lowest p-value obtained for the case of arthritis treatment (p-value: 0.06). In this case, however, our estimated coefficients have the expected sign, indicating a higher take-up of medication treatment among respondents in the 2nd to 4th quartile relative to the bottom $25 \%$ of the income distribution.

Also, the evidence regarding any important wealth gradients is weak at best. Our set of wealth controls does not enter any model with statistical significance, with the exception of hypertension ( $\mathrm{p}$-value: 0.0273 ). This rejection, however, is entirely due to the significantly lower take-up rates of respondents in the 2nd quartile of the wealth distribution relative to the omitted categories (the 1st quartile).

So far, the disease-specific approach has only delivered mixed evidence for socioeconomic inequity in medication treatment, with some indication for small effects of city size and education. Our final set of controls relating to the respondents' insurance status, however, displays an 
extremely coherent pattern across all disease categories. Having any health insurance coverage always increases the probability of medication treatment take-up statistically significantly, and often with very sizable effects. Specifically, our point estimates for the partial effects of having any health insurance coverage on indicated drug use are 11.7 percentage points for arthritis, 3.4 percentage points for diabetes, 22.7 percentage points for heart attack, 11.9 percentage points for hypertension, 7.8 percentage points for respiratory diseases and 13.4 percentage points for stroke. Thus, the size of the estimated effects of insurance status clearly dominate those of all other enabling factors, in addition to being estimated fairly precisely. Furthermore, the disease-specific models also confirm our previous evidence regarding the effects of multiple health insurance coverage. Having the right to medical attention under more than one scheme does not seem to increase the probability of medication treatment any further. Its estimated partial effects are of varying sign and remain statistically insignificant throughout.

\subsection{Regressions with Detailed Insurance Controls}

Given the central importance of health insurance coverage for the take-up of drug treatment and its clear dominance relative to the other enabling factors, we scrutinize the effects of insurance coverage further by re-running all of our full models, replacing the simple binary insurance indicator by a more detailed set of dummy variables that also indicate the respective source of coverage. Table V presents the estimated partial effects of these detailed insurance controls for all of these regressions. Although the relatively low prevalence of some of the insurance categories such as NAVY/DEFENCE/PEMEX, private insurance and other insurance makes it fairly challenging to obtain precise estimates for these insurance types, a remarkably consistent picture emerges: Having health insurance coverage leads to higher take-up rates of indicated medication treatment, largely irrespective of the exact type of coverage. Particularly, whenever statistically significant, the impact of all types of health insurance coverage on medication take-up is positive. Note only their sign, but also the size of the estimated partial effects is remarkable similar across insurance types. Specifically, associated Wald-tests for the null hypothesis of "identical partial effects for all insurance categories" always fail to reject at any conventional level of significance. Thus, although our aggregate insurance indicator gives clearly the most weight to the largest provider, the IMSS, our disaggregated analysis shows that any form of coverage is beneficial for the take-up of medication treatment, with fairly large and often significant positive effects on the probability of indicated medication use. At the same time, having coverage under more than one scheme, does not seem to increase the probability of take-up any further. The estimated partial effects for multiple coverage usually feature the opposite sign, but a similar magnitude to the insurance indicators, implying no additional effect of multiple coverage on take-up. 


\section{Discussion and Conclusion}

Our study investigated the role of various dimensions of socioeconomic status for the needadjusted take-up of medication treatment among older Mexicans with specific health conditions. We firstly identified strong bivariate socioeconomic gradients in need-adjusted medication use for city size, education, income, wealth and health insurance status, with higher take-up rates among more urban and better off respondents. Yet, estimation of a full model featuring all socioeconomic controls revealed health insurance coverage as the clearly dominating factor for explaining medication treatment take-up. Health insurance status consistently emerged as the most important enabling factor for take-up in a variety of models, whether estimated on pooled data or disease-specific estimation samples. The corresponding effects of insurance coverage on medication use are thereby fairly sizable. Specifically, having any from of coverage leads to increased take-up rates that range from 3.4 percentage points for the treatment of diabetes to more than 20 percentage points for the management of postmyocardial infarction. Moreover, the strong positive effects of insurance coverage were not limited to beneficiaries of the largest social insurer, the IMSS. Rather any type of health insurance coverage was found to strongly affect the take-up of indicated medication treatment.

While we deem our results most conclusive with respect to the beneficial effects of insurance coverage on drug use, a potential caveat regarding our need-adjustment may have led to some underestimation of the effects of all our enabling factors. Particularly, any preexisting gradients in access to general health care, testing or screening might affect the availability of a diagnosis and thereby our sample selection, which may result in an underestimation of any socioeconomic gradients in medication treatment. Given the interpretation of our estimates as lower bounds, we conclude that health insurance status is clearly a highly important enabling factor for medication use, without fully dismissing any potential role for other enabling factors. Particularly, access to health infrastructure and human capital may also be critical determinants of treatment take-up, and we have presented some evidence regarding their potential importance, even if it is not fully conclusive.

Overall, our results have important policy implications. Particularly, we have shown that self-medication is likely to be a fairly imperfect substitute for formal care with respect to promoting rational medication use. Despite its disproportionately high occurrence among individuals with low socioeconomic status and lack of insurance coverage (Pagán et al., 2006), self-medication does not seem to be able to eliminate socioeconomic gradients in indicated medication use. Rather, access to reliable and responsive health care providers coupled with appropriate drug benefits seems to be key for enhancing the take-up of medication treatment among individuals in need. Thus, expanding coverage to the uninsured population appears to be the most promising health policy response for ensuring rational medication use. Within the Mexican context, we therefore also expect the Seguro Popular to have a large positive effect on medication treatment take-up among the newly covered. Thus, apart from any added financial protection, the Seguro Popular also seems to be a promising intervention for reducing health care utilization disparities, such as socioeconomic gradients in medication use. 


\section{References}

Andersen R. 1968. Behavioral model of families' use of health services. Research Series No. 25, Center for Health Administration Studies, University of Chicago, Chicago.

Andersen R. 1995. Revisiting the behavioral model of access to medical care: Does it matter? Journal of Health and Social Behavior 36:1-10.

Barraza-Lloréns M, Bertozzi S, Gonzáez-Pier E, Gutiérrez J. 2002. Addressing inequity in health and health care in Mexico, Health Affairs 21:47-56.

Behrman J, Behrman J, Perez N. 2006. Where Should UNU-WIDER Place Their Bets? Some Considerations for Prioritizing Future Economic Research on Health and Development, presented at the UNU-WIDER conference on "Advancing Health Equity", Helsinki 2006.

Case A, le Roux I, Menendez A. 2004. Medical compliance and income-health gradients. American Ecnonomic Review Papers and Proceedings 94: 331-335.

Cetrángolo O, Cruces G, Titelman D. 2006. Social protection and health systems in Latin America and the Caribbean. Economic Commission for Latin America and the Caribbean, Santiago de Chile.

Danzon P, Furukawa M. 2003. Prices and availability of pharmaceuticals: Evidence from nine countries. Health Affairs (Web Exclusive) W3: 521-526.

Gakidou E, Lozano R, González-Pier E, Abbott-Klafter J, Barofsky JT, Bryson-Cahn C, Feehan DM, Lee DK, Hernández-Llamas H, Murray CJ. 2006. Assessing the effect of the 2001-06 Mexican health reform: an interim report card. The Lancet 368:1920-1935.

Goldman D, Smith J. 2002. Can patient self-management help explain the SES health gradient. Proceedings of the National Academy of Sciences of the United States of America 99: 10929-34.

Goldman D, Smith J. 2005. Socioeconomic differences in the adoption of new medical technologies. NBER Working Paper W11218, Cambridge, MA.

González-Pier E, Gutiérrez-Delgado C, Stevens G, Barraza-Lloréns M, Porras-Condey R, Carvalho, N, Loncich K, Dias R, Kulkarni S, Casey A, Murakami Y, Ezzati M, Salomon J. 2006. Priority setting for health interventions in Mexico's System of Social Protection in Health. The Lancet 368: 1608-1618.

Jacobzone S. 2003. Introduction to the ageing-related diseases project, in: OECD: A diseasebased comparison of health systems: What is best and at what cost? Paris: OECD,11-27.

Jamison D, Breman J, Measham A, Alleyne G, Claeson M, Evans D, Jha P, Mills A, Musgrove P, (eds.). 2006a. Priorities in Health, New York: Oxford University Press and The World Bank. 
Jamison D, Breman J, Measham A, Alleyne G, Claeson M, Evans D, Jha P, Mills A, Musgrove P, (eds.). 2006b. Disease Control Priorities in Developing Countries (2nd Edition), New York: Oxford University Press and The World Bank.

Knaul F, Frenk J. 2005. Health insurance in Mexico: Achieving universal coverage through structural reform. Health Affairs 24: 1467-1476.

Lleras-Muney A, Lichtenberg F. 2002. The effect of education on medical technology adoption: Are the more educated more likely to use new drugs. NBER Working Paper No. W9185, Cambridge, MA.

Lopez A, Mathers C, Ezzati M, Jamison D, and Murray C. 2006. Global Burden of Disease and Risk Factors. New York: Oxford University Press and the World .Bank.

Merino-Juárez M, Alarcón-Gómez M, Lozano-Ascencio R. 2004. SHA-based health accounts in 13 OECD countries: Country studies: Mexico: National health accounts 2001, Paris: OECD.

Ministry of Health. 2005. Hacia una política farmacéutica integral para México. Mexico City: Ministry of Health.

Moise P, Docteur E. 2007. Pharmaceutical pricing and reimbursement policies in Mexico, OECD Health Working Paper 25.

OECD. 2005a. OECD review of health systems - Mexico, Paris: OECD.

OECD. 2005b. OECD Health data 2005, Paris: OECD.

PAHO. 2002. Mortality data 2002. Washington DC: PAHO.

Pagán J, Ross S, Yau J, Polsky D. 2006. Self-medication and health insurance coverage in Mexico, Health Policy 75:170-77.

Pagán, J, Puig, A, Soldo, B. 2007. Health insurance coverage and the use of preventive care services by Mexican adults, Health Economics, forthcoming.

Palloni A, Soldo B, Wong R, Eniry M. 2004. Health status in a national sample of elderly Mexicans, University of Wisconsin, Madison.

Perry G, Maloney W, Arias O, Fajnzylber P, Mason A, Saavedra-Chanduvi J. 2007. Informality: Exit and exclusion. Washington: The World Bank.

Wagstaff A, van Doorslaer E. 2000. Equity in health care finance and delivery, Chapter 34 in: A. Cluyer, J. Newhouse (eds.): Handbook of Health Economics, Amsterdam Elsevier Science, 1803-62.

WHO. 2000. The benefits and risks of self-medication. WHO Drug Information 14: 1-2. 
Wong R, Espinoza M. 2004. Imputation of non-response on economic variables in the Mexican Health and Aging Study (MHAS/ENASEM) 2001, Population Studies Center, University of Pennsylvania, Philadelphia. 


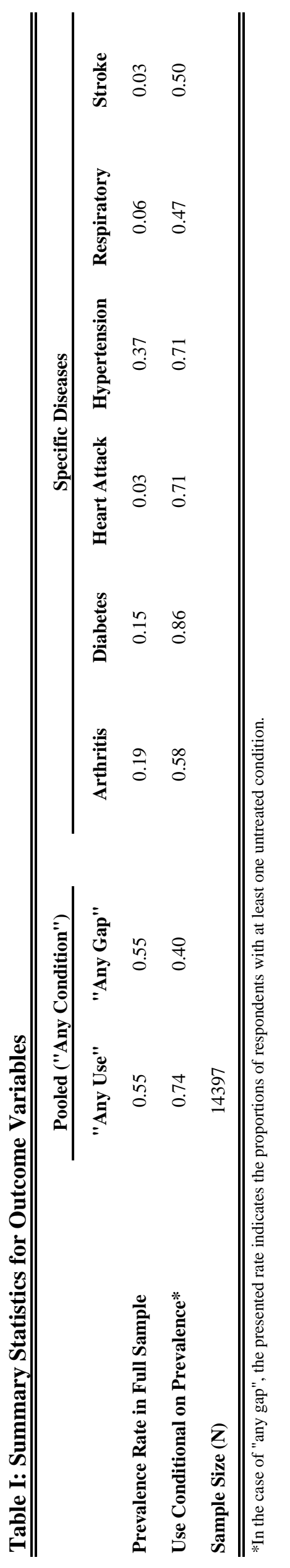




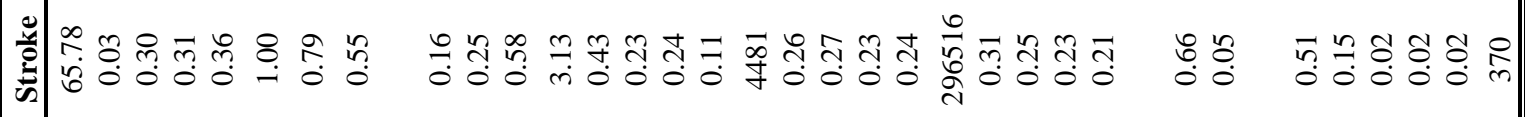

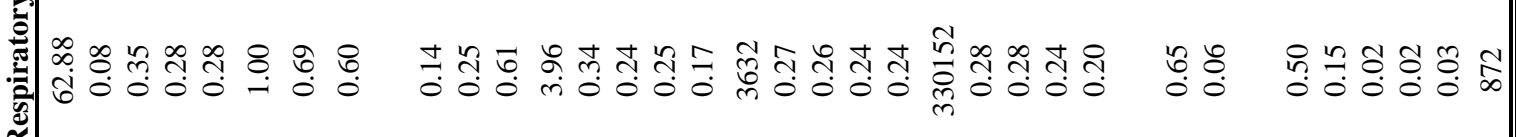

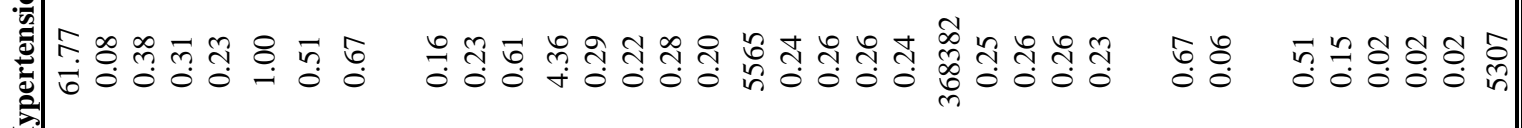

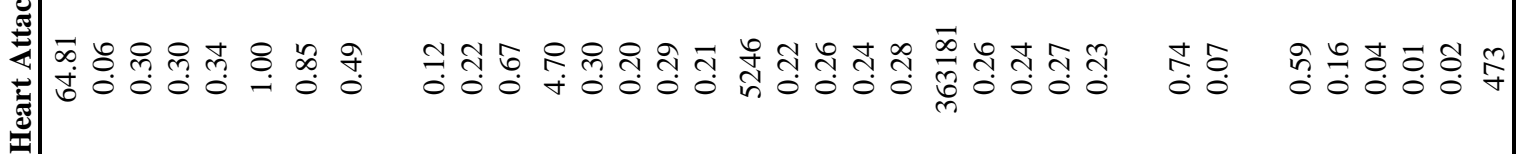

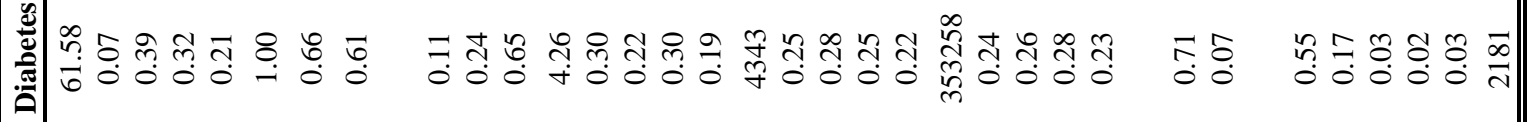

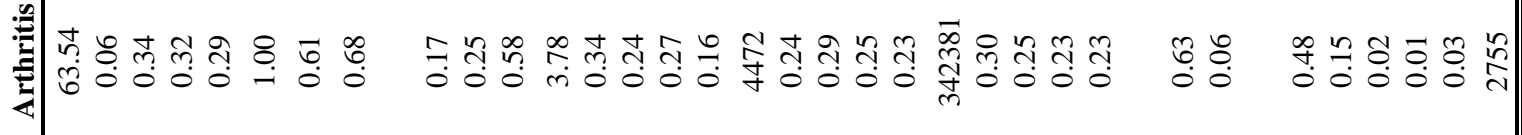

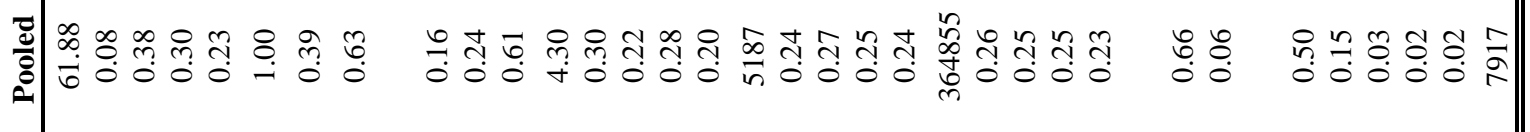

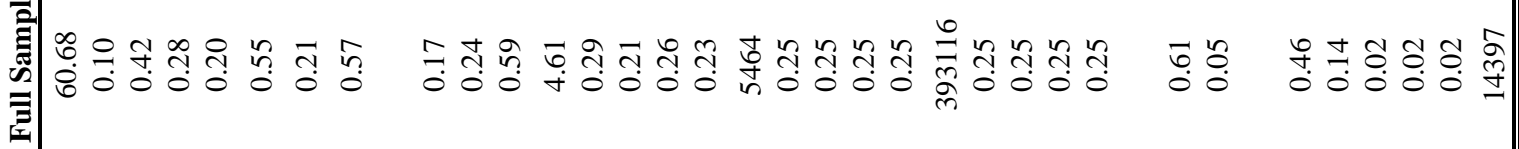

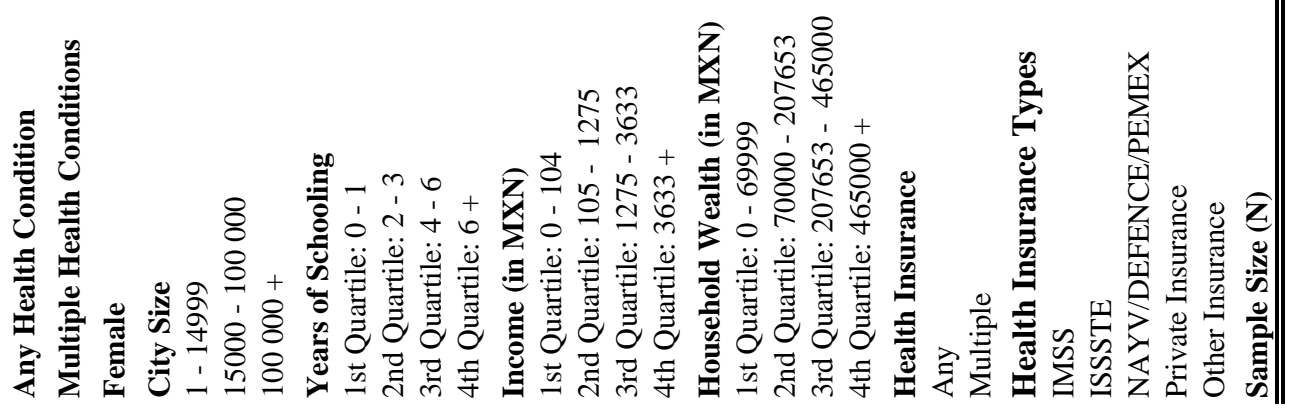




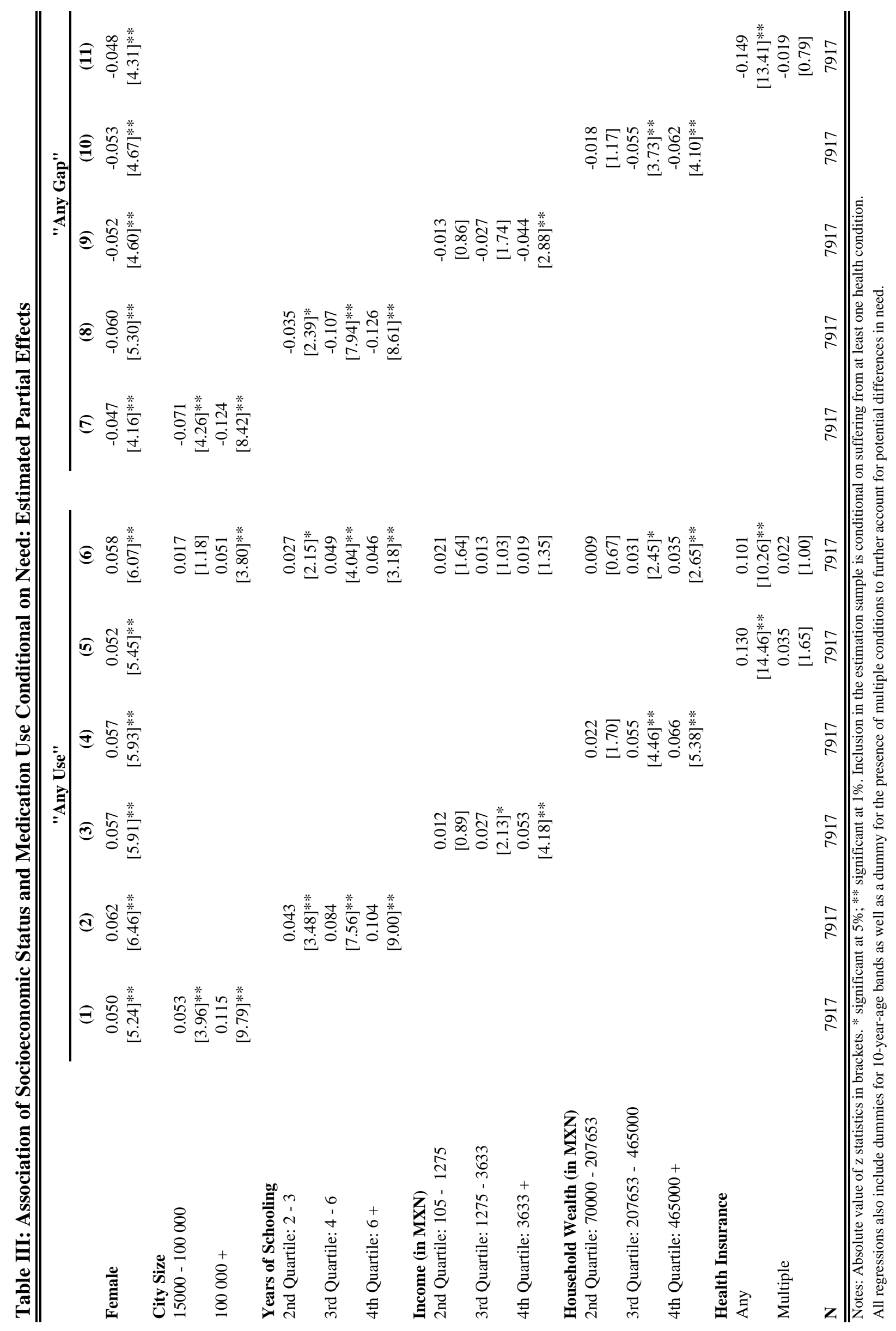




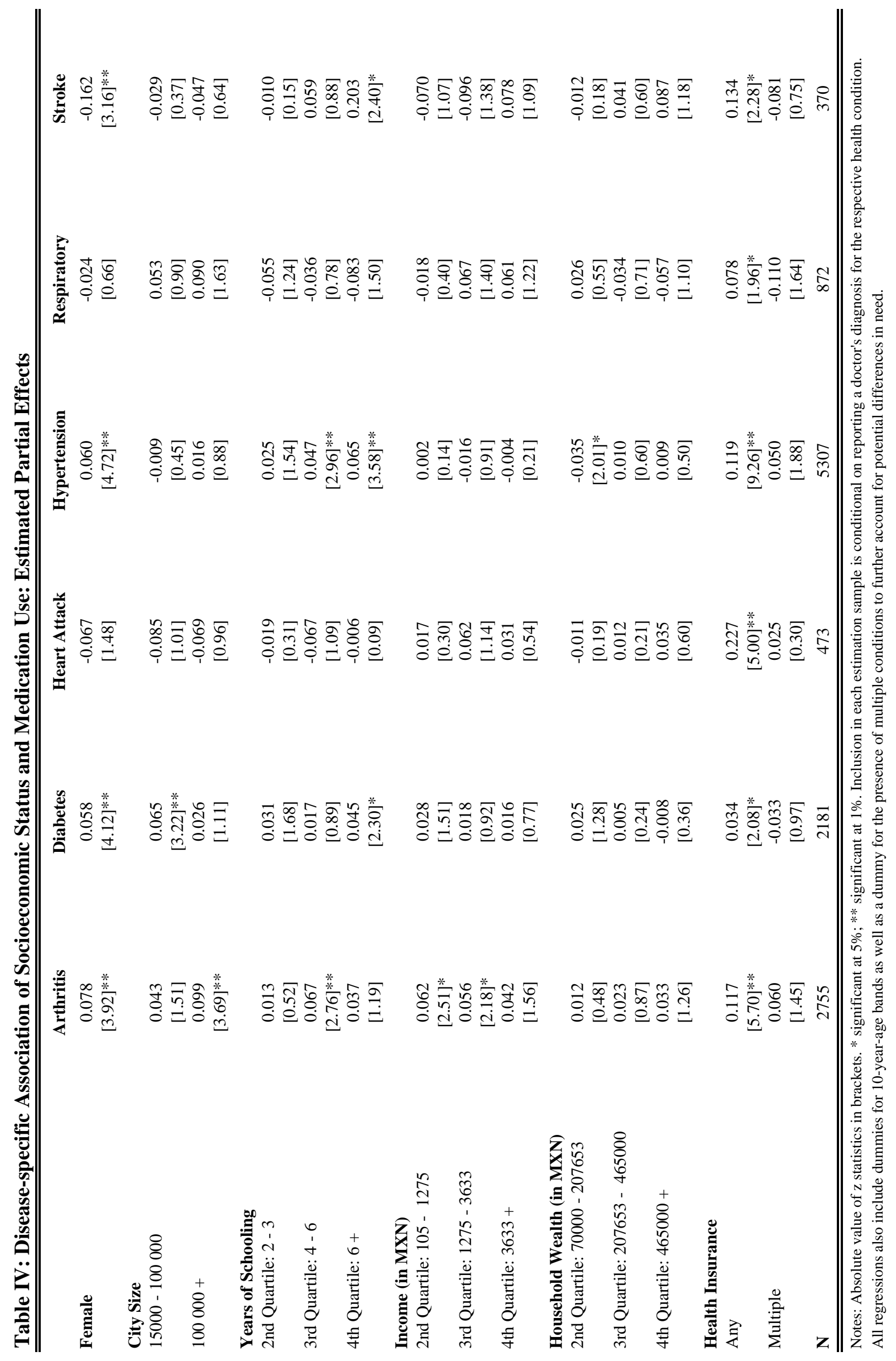




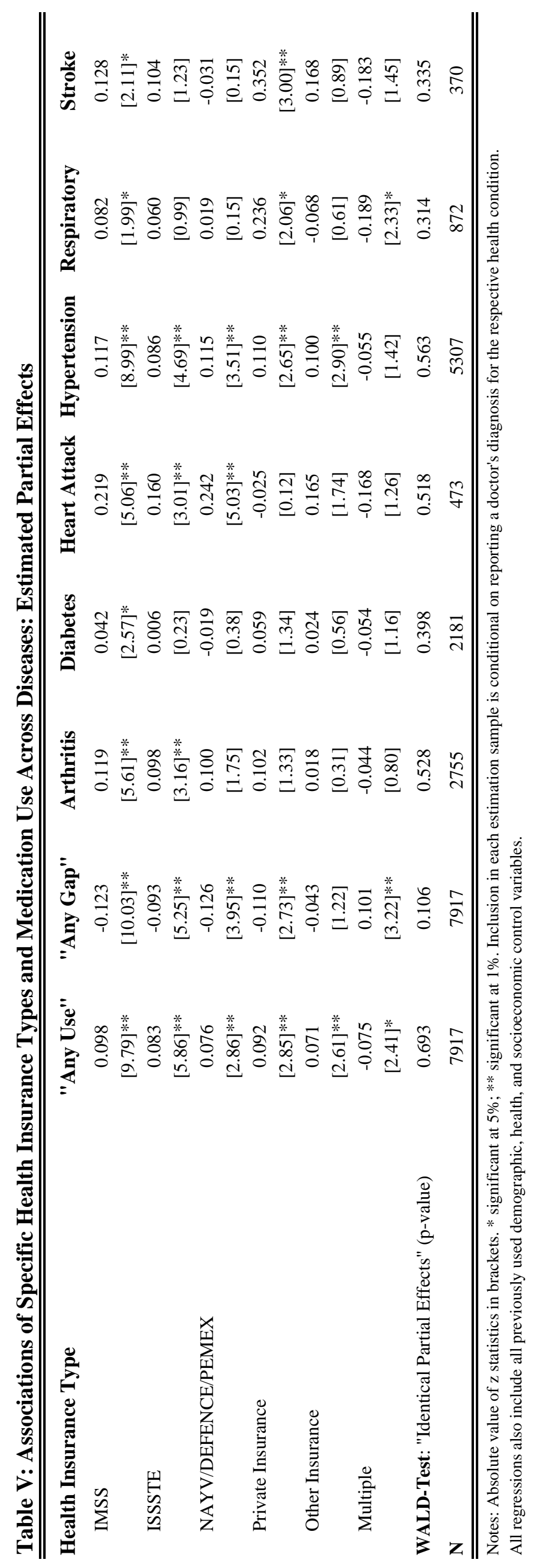




\section{Discussion Paper Series}

Mannheim Research Institute for the Economics of Aging Universität Mannheim

To order copies, please direct your request to the author of the title in question.

\begin{tabular}{|c|c|c|c|}
\hline Nr. & Autoren & Titel & Jahr \\
\hline $131-07$ & $\begin{array}{l}\text { Florian Heiss, Axel } \\
\text { Börsch-Supan, Michael } \\
\text { Hurd, David Wise }\end{array}$ & $\begin{array}{l}\text { Pathways to Disability: Predicting Health } \\
\text { Trajectories }\end{array}$ & 07 \\
\hline $132-07$ & Axel Börsch-Supan & Rational Pension Reform & 07 \\
\hline $133-07$ & Axel Börsch-Supan & Über selbststabilisierende Rentensysteme & 07 \\
\hline $134-07$ & $\begin{array}{l}\text { Axel Börsch-Supan, } \\
\text { Hendrik Jürges }\end{array}$ & $\begin{array}{l}\text { Early Retirement, Social Security and Well- } \\
\text { Being in Germany }\end{array}$ & 07 \\
\hline $135-07$ & Axel Börsch-Supan & Work Disability, Health, and Incentive Effects & 07 \\
\hline $136-07$ & $\begin{array}{l}\text { Axel Börsch-Supan, } \\
\text { Anette Reil-Held, } \\
\text { Daniel Schunk }\end{array}$ & $\begin{array}{l}\text { The savings behaviour of German households: } \\
\text { First Experiences with state promoted private } \\
\text { pensions }\end{array}$ & 07 \\
\hline $137-07$ & $\begin{array}{l}\text { Hendrik Jürges, } \\
\text { Mauricio Avendano, } \\
\text { Johan Mackenbach }\end{array}$ & $\begin{array}{l}\text { How comparable are different measures of self- } \\
\text { rated health? Evidence from five European } \\
\text { countries }\end{array}$ & 07 \\
\hline $138-07$ & $\begin{array}{l}\text { Hendrik Jürges, } \\
\text { Kerstion Schneider }\end{array}$ & $\begin{array}{l}\text { What can go wrong will go wrong: Birthday } \\
\text { effects and early tracking in the German school } \\
\text { system }\end{array}$ & 07 \\
\hline $139-07$ & Hendrik Jürges & $\begin{array}{l}\text { Does ill health affect savings intentions? } \\
\text { Evidence from SHARE }\end{array}$ & 07 \\
\hline $140-07$ & Hendrik Jürges & $\begin{array}{l}\text { Health inequalities by education, income, and } \\
\text { wealth: a comparison of } 11 \text { European countries } \\
\text { and the US }\end{array}$ & 07 \\
\hline 141-07 & Hendrik Jürges & $\begin{array}{l}\text { Healthy minds in healthy bodies. An } \\
\text { international comparison of education-related } \\
\text { inequality in physical health among older adults }\end{array}$ & 07 \\
\hline $142-07$ & $\begin{array}{l}\text { Karsten Hank, } \\
\text { Stephanie Stuck }\end{array}$ & $\begin{array}{l}\text { Volunteer Work, Informal Help, and Care among } \\
\text { the 50+ in Europe: Further Evidence for 'Linked' } \\
\text { Productive Activities at Older Ages }\end{array}$ & 07 \\
\hline 143-07 & Jürgen Maurer & $\begin{array}{l}\text { Assessing Horizontal Equity in Medication } \\
\text { Treatment Among Elderly Mexicans: Which } \\
\text { Socioeconomic Determinants Matter Most? }\end{array}$ & 07 \\
\hline
\end{tabular}

